\title{
TENSOR PRODUCT BASES AND TENSOR DIAGONALS
}

\author{
BY \\ J. R. HOLUB( ${ }^{(1)}$
}

\begin{abstract}
Let $X$ and $Y$ denote Banach spaces with bases $\left(x_{i}\right)$ and $\left(y_{i}\right)$, respectively, and let $X \otimes_{\delta} Y$ and $X \otimes_{\pi} Y$ denote the completion in the $\varepsilon$ and $\pi$ crossnorms of the algebraic tensor product $X \otimes Y$.

The purpose of this paper is to study the structure of the tensor product spaces $X \otimes_{8} Y$ and $X \otimes_{\pi} Y$ through a consideration of the properties of the tensor product basis $\left(x_{i} \otimes y_{j}\right)$ for these spaces and the tensor diagonal $\left(x_{i} \otimes y_{i}\right)$ of such bases.
\end{abstract}

1. Introduction. Let $X$ and $Y$ be Banach spaces and denote by $X \otimes_{\varepsilon} Y$ and $X \otimes_{\pi} Y$ the completion of the algebraic tensor product of $X$ and $Y$ in the $\varepsilon$ and $\pi$ topologies respectively (see $\S 2$ for definitions). If $\left(x_{i}\right)$ is a basis for $X$ and $\left(y_{i}\right)$ a basis for $Y$, then the sequence of tensors $\left(x_{i} \otimes y_{j}\right)$, ordered in a certain fashion, is a basis for both $X \otimes_{\varepsilon} Y$ and $X \otimes_{\pi} Y$ and is called the tensor product basis. We call the subsequence $\left(x_{i} \otimes y_{i}\right)$ of $\left(x_{i} \otimes y_{j}\right)$ the tensor diagonal of the bases $\left(x_{i}\right)$ and $\left(y_{i}\right)$.

The purpose of this paper is to study the properties of tensor product bases and tensor diagonals and thereby also to study the structure of the spaces $X \otimes_{\varepsilon} Y$ and $X \otimes_{\pi} Y$.

In $\$ 3$ and $\$ 4$ the permanence properties of tensor product bases and tensor diagonals in the $\varepsilon$ and $\pi$ topologies are discussed.

In $\$ 5$ conditions on the bases $\left(x_{i}\right)$ and $\left(y_{i}\right)$ which are sufficient to force the diagonal $\left(x_{i} \otimes y_{i}\right)$ to be similar to a given basis are determined. A complete characterization of the diagonals of the unit vector bases in $l^{p} \otimes_{\varepsilon} l^{r}$ is obtained.

2. Preliminary results and notation. The only spaces considered in this paper will be Banach spaces. If $X$ is a given space we will denote its dual or conjugate space by $X^{*}$. The closed linear span of a sequence $\left(x_{i}\right)$ in $X$ is denoted by $\left[x_{i}\right]$.

A sequence $\left(x_{i}\right)$ in $X$ is called a basis for $X$ (basic sequence in $X$ ) if for each $x$ in $X$ (for each $x$ in $\left[x_{i}\right]$ ) there exists a unique sequence of scalars $\left(a_{i}\right)$ such that $x=\sum_{i=1}^{\infty} a_{i} x_{i}$, convergence in the norm topology of $X$. If for each $x$ in $X$ (for each $x$ in $\left[x_{i}\right]$ ) this convergence is unconditional, then $\left(x_{i}\right)$ is called an unconditional basis (unconditional

Received by the editors September 29, 1969 and, in revised form, January 5, 1970.

AMS 1969 Subject Classifications. Primary 4620, 4610; Secondary 4601. diagonal.

Key Words and Phrases. Tensor product, Schauder basis, tensor product basis, tensor

(1) This paper is part of the author's doctoral dissertation written at Louisiana State University under the direction of Professor J. R. Retherford. The author wishes to thank Professor Retherford for his advice and encouragement in the preparation of this paper.

Copyright (c) 1970, American Mathematical Society 
basic sequence). For a discussion of unconditional convergence in Banach spaces see [14].

Associated with a basis $\left(x_{i}\right)$ in $X$ is a sequence of linear functionals $\left(f_{i}\right)$ in $X^{*}$ defined by $f_{n}(x)=f_{n}\left(\sum_{i} a_{i} x_{i}\right)=a_{n}$ and called the associated sequence of coefficient functionals. A basis $\left(x_{i}\right)$ having coefficient functionals $\left(f_{i}\right)$ is denoted by $\left(x_{i}, f_{i}\right)$. It is well known that if $\left(x_{i}, f_{i}\right)$ is a (unconditional) basis for $X$ then $\left(f_{i}\right)$ is a (unconditional) basic sequence in $X^{*}$.

A basis $\left(x_{i}\right)$ is called seminormalized if $0<\inf _{i}\left\|x_{i}\right\| \leqq \sup _{i}\left\|x_{i}\right\|<+\infty$. Throughout this paper, unless specific mention is made to the contrary, all bases will be assumed to be seminormalized.

The following internal characterization of bases is due to M. M. Grinblyum [9] and is called the " $K$-condition".

THEOREM. Let $\left(x_{i}\right)$ be a sequence in $X$ such that $\left[x_{i}\right]=X$. Then $\left(x_{i}\right)$ is a basis for $X$ if and only if there exists $a K \geqq 1$ such that for all $p \leqq q$ and all sequences $\left(a_{i}\right)$, $\left\|\sum_{i=1}^{p} a_{i} x_{i}\right\| \leqq K\left\|\sum_{i=1}^{q} a_{i} x_{i}\right\|$.

In the case $K=1,\left(x_{i}\right)$ is called a monotone basis.

A similar criterion called the unconditional form of the $K$-condition characterizes unconditional bases. One has only to replace initial segments in the above theorem with arbitrary finite sets of integers.

If $\left(x_{i}, f_{i}\right)$ is a basis for $X$ and $\left(y_{i}, g_{i}\right)$ is a basis for $Y$, then $\left(x_{i}\right)$ and $\left(y_{i}\right)$ are said to be similar if $\sum_{i} a_{i} x_{i}$ converges if and only if $\sum_{i} a_{i} y_{i}$ converges. It is a consequence of the Banach-Steinhaus theorem and the open mapping theorem that the bases $\left(x_{i}\right)$ and $\left(y_{i}\right)$ are similar if and only if there exists a linear homeomorphism $T: X \rightarrow Y$ such that $T\left(x_{i}\right)=y_{i}$.

It follows from the norm-determining property of $\left[f_{i}\right]$ over $X$ [21] that the sequence of coefficient functionals $\left(g_{i}\right)$ in $\left[f_{i}\right]^{*}$ associated with the basic sequence $\left(f_{i}\right)$ is similar to the basis $\left(x_{i}\right)$. Hence we write $\left(f_{i}, x_{i}\right)$ is a basic sequence in $X^{*}$.

Let $\left(x_{i}, f_{i}\right)$ be a basis for $X$. Then $\left(x_{i}\right)$ is said to be

(i) shrinking [4, p. 69] if $\left(f_{i}\right)$ is a basis for $X^{*}$ (in the norm topology on $X^{*}$ ),

(ii) boundedly complete $\left[4\right.$, p. 69] if $\sup _{n}\left\|\sum_{i=1}^{n} a_{i} x_{i}\right\|<+\infty$ implies $\sum_{i} a_{i} x_{i}$ converges,

(iii) of type $\mathrm{wc}_{0}$ [7] if $\left\{x_{i}\right\}$ converges weakly to zero, (this property has also been called semishrinking [15], [17]). (Note: we denote weak convergence of a sequence $\left(x_{n}\right)$ to $x$ by $x_{n} \stackrel{w}{\rightarrow} x$.)

(iv) of type $\operatorname{swc}_{0}$ [7] if there is a subsequence $\left(x_{n_{i}}\right)$ of $\left(x_{i}\right)$ such that $x_{n_{i}} \stackrel{w}{\rightarrow} 0$.

(v) of type $\left(\mathrm{wc}_{0}\right)^{*}$ [7] if $f_{i} \stackrel{w}{\rightarrow} 0$,

(vi) of type $\mathrm{P}[20]$ if $\sup _{n}\left\|\sum_{i=1}^{n} x_{i}\right\|<+\infty$,

(vii) of type $\mathrm{P}^{*}[20]$ if there is an $f$ in $X^{*}$ such that $f\left(x_{i}\right)=1$ for all $i$.

Throughout the paper the notation $X=Y$ will mean $X$ is linearly homeomorphic (isomorphic) to $Y$ and $X \subset Y$ will mean $X$ is isomorphic to a closed subspace of $Y$.

Finally, $B(X, Y)$ denotes the bounded bilinear forms on $X \times Y$ and $\mathscr{L}(X, Y)$ 
denotes the bounded linear operators from $X$ to $Y$. The sequence $\left(e_{i}\right)$ will always denote the usual unit vector basis for one of the spaces $c_{0}$ or $l^{p}(1 \leqq p<+\infty)$.

Let $X$ and $Y$ be Banach spaces. We will denote by $X \otimes_{\varepsilon} Y$ the completion of the algebraic tensor product $X \otimes Y$ in the norm

$$
\left\|\sum_{i=1}^{n} x_{i} \otimes y_{i}\right\|=\sup _{\|f\| \leqq 1, f \in X^{*}:\|g\| \leqq 1, g \in Y^{*}}\left|\sum_{i=1}^{n} f\left(x_{i}\right) g\left(y_{i}\right)\right|,
$$

and we denote by $X \otimes_{\pi} Y$ the completion of $X \otimes Y$ in the norm

$$
\left\|\sum_{i=1}^{n} x_{i} \otimes y_{i}\right\|=\inf \left\{\sum_{j=1}^{k}\left\|x_{j}^{\prime}\right\|\left\|y_{j}^{\prime}\right\|: \sum_{j=1}^{k} x_{j}^{\prime} \otimes y_{j}^{\prime}=\sum_{i=1}^{n} x_{i} \otimes y_{i}\right\} .
$$

A crossnorm $\alpha$ on a tensor product space is one for which $\alpha(x \otimes y)=\|x\|\|y\|$. A crossnorm $\alpha$ is said to be uniform [19, p. 9] if for any bounded linear operators $T$ on $X$ and $S$ on $Y$,

$$
\alpha\left(\sum_{i} S\left(x_{i}\right) \otimes T\left(y_{i}\right)\right) \leqq\|S\|\|T\| \alpha\left(\sum_{i} x_{i} \otimes y_{i}\right) .
$$

It is well known that both $\varepsilon$ and $\pi$ are uniform crossnorms.

The following results on tensor products will be used frequently, most often without specific reference.

(1) If $M$ is a closed subspace of $X$ and $N$ is a closed subspace of $Y$, then $M \otimes_{\varepsilon} N$ is a closed subspace of $X \otimes_{\varepsilon} Y[19$, p. 35]. As we have mentioned above, we will denote this by $M \otimes_{\varepsilon} N \subset X \otimes_{\varepsilon} Y$.

The proposition obtained by replacing $\varepsilon$ by $\pi$ in (1) is in general not true [19, p. 35].

(2) $X^{*} \otimes_{\varepsilon} Y^{*} \subset\left(X \otimes_{\pi} Y\right)^{*}[19$, p. 34].

(3) The space $\left(X \otimes_{\pi} Y\right)^{*}$ has a representation as the space of all continuous linear maps from $X$ to $Y^{*}$; i.e. $\left(X \otimes_{\pi} Y\right)^{*}=\mathscr{L}\left(X, Y^{*}\right)[19$, p. 45].

(4) The space $\left(X \otimes_{\varepsilon} Y\right)^{*}$ consists exactly of those $v$ in $B(X, Y)$ that can be represented in the form

$$
v(w)=\int_{S \times T} w_{0}\left(x^{\prime}, y^{\prime}\right) d \mu\left(x^{\prime}, y^{\prime}\right)
$$

where $S$ and $T$ are suitable closed equicontinuous subsets of $X_{\sigma}^{*}$ and $Y_{\sigma}^{*}$ and where $w_{0}$ is the restriction of the bilinear form $w$ on $X^{*} \times Y^{*}$ to $S \times T$ [18, p. 168].

(5) If $X^{*}$ has a basis then $\left(X \otimes_{\varepsilon} Y\right)^{*}=X^{*} \otimes_{\pi} Y^{*}[12]$.

(6) If $\left(x_{i}, f_{i}\right)$ is a basis for $X$ and $\left(y_{i}, g_{i}\right)$ is a basis for $Y$ then the sequence $\left(x_{i} \otimes y_{j}\right)$ in $X \otimes Y$ ordered in the following fashion

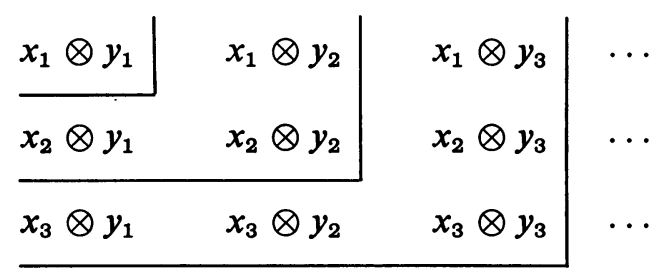


is $a$ basis for both $X \otimes_{\varepsilon} Y$ and $X \otimes_{\pi} Y$ and the sequence of coefficient functionals associated with $\left(x_{i} \otimes y_{j}\right)$ is the sequence $\left(f_{i} \otimes g_{j}\right)$ [8].

We call the basis $\left(x_{i} \otimes y_{j}\right)$ the tensor product of the bases $\left(x_{i}\right)$ and $\left(y_{i}\right)$, or the tensor product basis.

The subsequence $\left(x_{i} \otimes y_{i}\right)$ of the basis $\left(x_{i} \otimes y_{j}\right)$ will be called the tensor diagonal of the bases $\left(x_{i}\right)$ and $\left(y_{i}\right)$.

3. The $\varepsilon$-tensor product. In this section we will be concerned mainly with determining those properties of the bases $\left(x_{i}\right)$ and $\left(y_{i}\right)$ which carry over to the basis $\left(x_{i} \otimes y_{j}\right)$ or to the tensor diagonal $\left(x_{i} \otimes y_{i}\right)$ in $X \otimes_{\varepsilon} Y$. We will also deal with the converse problem.

We begin with several lemmas. The proofs are simple and will be omitted.

LEMMA 3.1. Let $\left(x_{i} \otimes y_{j}\right)$ be a tensor product basis for $X \otimes_{\alpha} Y$ where $\alpha$ is any crossnorm on $X \otimes Y$. Then $\left(x_{i} \otimes y_{1}\right)$ is similar to $\left(x_{i}\right)$ and $\left(x_{1} \otimes y_{i}\right)$ is similar to $\left(y_{i}\right)$.

LEMMA 3.2. Let $\left(x_{i}, f_{i}\right)$ be a shrinking basis for $X$. Then any subsequence $\left(x_{n_{i}}\right)$ is shrinking.

LEMMA 3.3. Let $\left(x_{i}\right)$ be a boundedly complete basis for $X$. Then any subsequence $\left(x_{n_{1}}\right)$ is boundedly complete.

Our first theorem is a consequence of Grothendieck's characterization of $\left(X \otimes_{8} Y\right)^{*}$ in the case where $X^{*}$ has a basis (see $\left.\S 2\right)$.

THEOREM 3.4. Let $\left(x_{i}\right)$ and $\left(y_{i}\right)$ be bases for $X$ and $Y$ respectively. Then the tensor product basis $\left(x_{i} \otimes y_{j}\right)$ for $X \otimes_{\varepsilon} Y$ is shrinking if and only if $\left(x_{i}\right)$ and $\left(y_{i}\right)$ are shrinking bases.

Proof. If $\left(x_{i} \otimes y_{j}\right)$ is shrinking then each of $\left(x_{i} \otimes y_{1}\right)$ and $\left(x_{1} \otimes y_{i}\right)$ is shrinking by Lemma 3.2. It follows from Lemma 3.1 that $\left(x_{i}\right)$ and $\left(y_{i}\right)$ are shrinking.

If $\left(x_{i}, f_{i}\right)$ and $\left(y_{i}, g_{i}\right)$ are both shrinking then by definition $\left(f_{i}\right)$ is a basis for $X^{*}$ and $\left(g_{i}\right)$ is a basis for $Y^{*}$. Since $\left(f_{i} \otimes g_{j}\right)$ is the sequence of coefficient functionals associated with the basis $\left(x_{i} \otimes y_{j}\right)$ and $\left(f_{i} \otimes g_{j}\right)$ is a basis for $X^{*} \otimes_{\pi} Y^{*}=\left(X \otimes_{\varepsilon} Y\right)^{*}$, the basis $\left(x_{i} \otimes y_{j}\right)$ is shrinking.

It follows from Theorem 3.4 and Lemma 3.2 that if $\left(x_{i}\right)$ and $\left(y_{i}\right)$ are shrinking bases for $X$ and $Y$ then the diagonal $\left(x_{i} \otimes y_{i}\right)$ is shrinking in $X \otimes_{\varepsilon} Y$. The next theorem shows that the same result is true assuming only that one of $\left(x_{i}\right)$ and $\left(y_{i}\right)$ is shrinking. It is also our first example (others will be seen later) of how strongly some properties of bases carry over to the tensor diagonal.

THEOREM 3.5. Let $\left(x_{i}, f_{i}\right)$ be a shrinking basis for $X$ and $\left(y_{i}, g_{i}\right)$ a basis for $Y$. Then the tensor diagonal in $X \otimes_{\varepsilon} Y$ is shrinking.

Proof. Let $\left(z_{n}\right)=\left(\sum_{j} b_{j}^{(n)} x_{j} \otimes y_{j}\right)$ be a bounded sequence in $\left[x_{i} \otimes y_{i}\right]$ with the property that $f_{k} \otimes g_{k}\left(z_{n}\right) \stackrel{n}{\rightarrow} 0$ for $k=1,2, \ldots$ That is, $b_{k}^{(n)} \stackrel{n}{\rightarrow} 0$ for $k=1,2, \ldots$ 
By virtue of the characterization of $\left(X \otimes_{\varepsilon} Y\right)^{*}$ given in $\S 2$, we need only show that

$$
\int_{S \times T}\left(\sum_{j=1}^{\infty} b_{j}^{(n)} x_{j} \otimes y_{j}\right)\left(x^{\prime}, y^{\prime}\right) d \mu\left(x^{\prime}, y^{\prime}\right) \stackrel{n}{\longrightarrow} 0
$$

for $S$ and $T$ equicontinuous subsets of $X_{\sigma}^{*}$ and $Y_{\sigma}^{*}[16]$.

Since $S \times T$ is a compact metric space [18, p. 87] and $z_{n}$ is in $C(S \times T)$ for each $n$ $[18$, p. 168$]$ it is sufficient to show that

$$
\sum_{j=1}^{\infty} b_{j}^{(n)} x_{j} \otimes y_{j}\left(x^{\prime} \otimes y^{\prime}\right)
$$

goes to zero with $n$ for each $\left(x^{\prime}, y^{\prime}\right)$ in $S \times T$ [6, p. 265].

To do this, fix $x^{\prime}$ in $S$ and $y^{\prime}$ in $T$. Then

$$
\sum_{j=1}^{\infty} b_{j}^{(n)} x_{j} \otimes y_{j}\left(x^{\prime} \otimes y^{\prime}\right)=x^{\prime}\left(\sum_{j=1}^{\infty} b_{j}^{(n)} y^{\prime}\left(y_{j}\right) x_{j}\right) .
$$

Now $f_{k}\left(\sum_{j} b_{j}^{(n)} y^{\prime}\left(y_{j}\right) x_{j}\right)=b_{k}^{(n)} y^{\prime}\left(y_{k}\right) \stackrel{n}{\rightarrow} 0$ for $k=1,2, \ldots$ since $\left(y_{k}\right)$ is a bounded set and we have seen that $b_{k}^{(n)} \stackrel{n}{\rightarrow} 0$. Therefore since $\left(x_{i}\right)$ is shrinking and the set $\left\{\sum_{j=1}^{\infty} b_{j}^{(n)} y^{\prime}\left(y_{j}\right) x_{j}\right\}$ is bounded in $X$ it follows that the sequence $\left\{\sum_{j=1}^{\infty} b_{j}^{(n)} y^{\prime}\left(y_{j}\right) x_{j}\right\}$ converges weakly to zero in $X$ [16]. In particular $x^{\prime}\left(\sum b_{j}^{(n)} y^{\prime}\left(y_{j}\right) x_{j}\right) \stackrel{n}{\rightarrow} 0$, and as we have noted above this implies $\left(x_{i} \otimes y_{i}\right)$ is shrinking.

Simple examples show that the tensor diagonal (and hence the tensor product) of boundedly complete bases need not be boundedly complete in $X \otimes_{8} Y$. However, in certain special cases this result is true.

Proposition 3.6. Let $\left(x_{i}\right)$ be a basis for a reflexive space $X$ and let $\left(y_{i}\right)$ be a boundedly complete basic sequence in $l^{1}$. Then the tensor diagonal $\left(x_{i} \otimes y_{i}\right)$ in $X \otimes_{\varepsilon}\left[y_{i}\right]$ is boundedly complete.

Proof. Suppose $\sup _{n}\left\|\sum_{i=1}^{n} a_{i} x_{i} \otimes y_{i}\right\|<+\infty$. Then

$$
\sup _{n} \sup _{\substack{\| f \underline{s}_{1} \\ f \in \mathbb{X}^{-1}}}\left\|\sum_{i=1}^{n} a_{i} f\left(x_{i}\right) y_{i}\right\|<+\infty,
$$

implying that $\sum_{i=1}^{\infty} a_{i} f\left(x_{i}\right) y_{i}$ converges for each $f$ in $X^{*}$. By the Banach-Steinhaus theorem the linear mapping $T: X^{*} \rightarrow\left[y_{i}\right] \subset l^{1}$ defined by $T(f)=\sum_{i=1}^{\infty} a_{i} f\left(x_{i}\right) y_{i}$ is continuous and therefore compact since $X^{*}$ is reflexive [6, p. 515]. But then the set $\left\{\sum_{i} a_{i} f\left(x_{i}\right) y_{i} \mid f \in X^{*},\|f\| \leqq 1\right\}$ is relatively compact in $\left[y_{i}\right]$ and so $\sum_{i=1}^{\infty} a_{i} f\left(x_{i}\right) y_{i}$ converges uniformly over $\|f\| \leqq 1$. It now follows by definition of the $\varepsilon$-norm that $\sum_{i=1}^{\infty} a_{i} x_{i} \otimes y_{i}$ converges in $X \otimes_{\varepsilon}\left[y_{i}\right]$ and $\left(x_{i} \otimes y_{i}\right)$ is boundedly complete.

THEOREM 3.7. Let $\left(x_{i}\right)$ be a basis for $X$ and $\left(y_{i}\right)$ a basis for $Y$. Then the basis $\left(x_{i} \otimes y_{i}\right)$ for $x \otimes_{\varepsilon} Y$ is of type $w_{0}$ if and only if each of $\left(x_{i}\right)$ and $\left(y_{i}\right)$ is of type $w_{0}$.

Proof. The proof of the first implication follows immediately from Lemma 1.1. The proof of the second is similar to that of Theorem 3.5. We give a brief sketch. 
Let $g$ be in $\left(X \otimes_{\varepsilon} Y\right)^{*}$. Then

$$
g(w)=\int_{S \times T} w_{0}\left(x^{\prime}, y^{\prime}\right) d \mu\left(x^{\prime}, y^{\prime}\right)
$$

where $S$ and $T$ are equicontinuous subsets of $X_{\sigma}^{*}$ and $Y_{\sigma}^{*}$ and $w_{0}$ is the restriction of the bilinear form $w$ on $X^{*} \times Y^{*}$ to $S \times T$ (recall that every $w$ in $X \otimes Y$ can be viewed as a bilinear form on $\left.X^{*} \times Y^{*}\right)$. Again, $S \times T$ is a metric space so since $x_{i} \otimes y_{j}\left(x^{\prime}, y^{\prime}\right)=x_{i}\left(x^{\prime}\right) y_{j}\left(y^{\prime}\right)$ for all $\left(x^{\prime}, y^{\prime}\right)$ in $S \times T$, and since by assumption $x_{i}\left(x^{\prime}\right) \stackrel{i}{\rightarrow} 0$ and $y_{j}\left(y^{\prime}\right) \stackrel{j}{\rightarrow} 0$, we have $\left(x_{i} \otimes y_{j}\right)$ (as a sequence in $C(S \times T)$ ) converges to zero at each point in $S \times T$ and hence converges weakly to zero in $C(S \times T)$.

Therefore

$$
g\left(x_{i} \otimes y_{j}\right)=\int_{S \times T} x_{i} \otimes y_{j}\left(x^{\prime}, y^{\prime}\right) d \mu\left(x^{\prime}, y^{\prime}\right)=\mu\left(x_{i} \otimes y_{j}\right) \rightarrow 0
$$

(since $\mu$ is in $\left.C(S \times T)^{*}\right)$ and $\left(x_{i} \otimes y_{j}\right)$ is of type $\mathrm{wc}_{0}$.

An inspection of the proof of Theorem 3.7 establishes the following result which should be compared with Theorem 3.5 .

THEOREM 3.8. Let $\left(x_{i}\right)$ be a basis of type $\mathrm{wc}_{0}$ for $X$ and let $\left(y_{i}\right)$ be any basis for $Y$. Then the tensor diagonal $\left(x_{i} \otimes y_{i}\right)$ in $X \otimes_{\varepsilon} Y$ is of type $\mathrm{wc}_{0}$.

CoRollary 3.9. Let $\left(x_{i}\right)$ and $\left(y_{i}\right)$ be bases for $X$ and $Y$ respectively. Then $\left(x_{i} \otimes y_{i}\right)$ is of type $\mathrm{swc}_{0}$ in $X \otimes_{\varepsilon} Y$ if and only if $\left(x_{i}\right)$ or $\left(y_{i}\right)$ is of type $\mathrm{swc}_{0}$.

Proof. Suppose $\left(x_{n_{i}}\right) \stackrel{w}{\rightarrow} 0$. By Theorem $3.8\left(x_{n_{i}} \otimes y_{n_{i}}\right) \stackrel{w}{\rightarrow} 0$ in $\left[x_{n_{i}}\right] \otimes_{\varepsilon}\left[y_{n_{i}}\right]$ $\subset X \otimes_{\varepsilon} Y$. Hence $\left(x_{i} \otimes y_{i}\right)$ is of type $\operatorname{swc}_{0}$.

Suppose $\left(x_{n_{i}} \otimes y_{n_{i}}\right) \stackrel{w}{\rightarrow} 0$ in $X \otimes_{\varepsilon} Y$. If $\left(y_{n_{i}}\right)^{w} \rightarrow 0$ there is an $f_{0}$ in $Y^{*}$ and a subsequence $\left(y_{m_{k}}\right)$ of $\left(y_{n_{i}}\right)$ such that $\left|f_{0}\left(y_{m_{k}}\right)\right| \geqq 1$ for $k=1,2, \ldots$ Since $\left(x_{m_{k}} \otimes y_{m_{k}}\right)$ $\stackrel{w}{\rightarrow} 0, f \otimes f_{0}\left(x_{m_{k}} \otimes y_{m_{k}}\right) \stackrel{k}{\rightarrow} 0$ for every $f$ in $X^{*}$ and so $\left|f\left(x_{m_{k}}\right)\right|\left|f_{0}\left(y_{m_{k}}\right)\right| \rightarrow 0$. It follows that $\left|f\left(x_{m_{k}}\right)\right| \stackrel{k}{\rightarrow} 0$ for each $f$ in $X^{*}$ and $\left(x_{i}\right)$ is of type $\operatorname{swc}_{0}$.

REMARK 3.10. The converse of Theorem 3.8 is false. In fact, there exist bases $\left(x_{i}\right)$ and $\left(y_{i}\right)$ for $C[0,1]$ neither of which is of type $w_{0}$, but whose diagonal $\left(x_{i} \otimes y_{i}\right)$ in $C[0,1] \otimes_{\varepsilon} C[0,1]$ is similar to the unit vector basis of $c_{0}[11]$.

In view of this situation the next proposition is interesting.

Proposition 3.11. Let $\left(x_{i}\right)$ be a basis for $X$. Then $\left(x_{i}\right)$ is of type $\mathrm{wc}_{0}$ if and only if the tensor diagonal $\left(x_{i} \otimes x_{i}\right)$ in $X \otimes_{\varepsilon} X$ is of type $\mathrm{wc}_{0}$.

Proof. The first implication follows immediately from Theorem 3.8.

Let $f$ be an element of $X^{*}$. Then $f \otimes f$ is in $\left(X \otimes_{\varepsilon} X\right)^{*}$ and $f \otimes f\left(x_{i} \otimes x_{i}\right)$ $=\left[f\left(x_{i}\right)\right]^{2}$. Since $\left(x_{i} \otimes x_{i}\right) \stackrel{w}{\rightarrow} 0$ we have $\left[f\left(x_{i}\right)\right]^{2} \rightarrow 0$ and $\left(x_{i}\right)$ is of type $\mathrm{wc}_{0}$.

Gelbaum and de Lamadrid have shown that the tensor product $\left(x_{i} \otimes y_{i}\right)$ of unconditional bases $\left(x_{i}\right)$ and $\left(y_{i}\right)$ need not be unconditional in either $X \otimes_{\varepsilon} Y$ or in $X \otimes_{\pi} Y$ [8].

In contrast to this result we prove the following theorem which illustrates again the permanence properties of the tensor diagonal. 
THEOREM 3.12. Let $\left(x_{i}\right)$ be an unconditional basis for $X,\left(y_{i}\right)$ a basis for $Y$, and $\alpha$ a uniform crossnorm. Then the tensor diagonal $\left(x_{i} \otimes y_{i}\right)$ in $X \otimes_{\alpha} Y$ is unconditional.

Proof. Suppose $\sum_{i=1}^{\infty} a_{i} x_{i} \otimes y_{i} \in X \otimes_{\alpha} Y$ and $\left(\varepsilon_{i}\right)$ is any \pm 1 sequence.

Then $\left(\varepsilon_{i} x_{i}\right)$ is a basis for $X$ similar to $\left(x_{i}\right)$, implying there exists a continuous linear mapping $T: X \rightarrow X$ for which $T x_{i}=\varepsilon_{i} x_{i}$. Now $\alpha$ is a uniform crossnorm so the tensor product map $T \otimes I: X \otimes_{\alpha} Y \rightarrow X \otimes_{\alpha} Y$ (where $I$ is the identity map) is continuous. It follows that

$$
T \otimes I\left(\sum_{i=1}^{\infty} a_{i} x_{i} \otimes y_{i}\right)=\sum_{i=1}^{\infty} \varepsilon_{i} a_{i} x_{i} \otimes y_{i}
$$

converges in $X \otimes_{\alpha} Y$. Since the sequence $\left(\varepsilon_{i}\right)$ was arbitrary, the basic sequence $\left(x_{i} \otimes y_{i}\right)$ is unconditional.

In particular, Theorem 3.12 holds when $\alpha=\varepsilon$ or $\alpha=\pi$ [19].

The converse to Theorem 3.12 in the case $X \otimes_{\varepsilon} Y$ is false, as is shown by the following example.

EXAMPLE 3.13. Let $\left(x_{i}\right)$ denote the conditional basis for $l^{1}$ defined by $x_{1}=e_{1}$, $x_{n}=-e_{n}+e_{n-1}$ for $n=2,3, \ldots$ Then the tensor diagonal $\left(x_{i} \otimes x_{i}\right)$ in $l^{1} \otimes_{\varepsilon} l^{1}$ is similar to the unit vector basis $\left(e_{i}\right)$ of $l^{1}$.

Proof. Suppose $\sum_{i=1}^{\infty} a_{i} x_{i} \otimes x_{i}$ is in $l^{1} \otimes_{\varepsilon} l^{1}$. Then $\sup _{n}\left\|\sum_{i=1}^{n} a_{i} x_{i} \otimes x_{i}\right\|_{\varepsilon} \leqq M$. From the definition of the $\varepsilon$-topology it follows that $\sup _{\|f\| \leqq 1}\left\|\sum_{i=1}^{n} a_{i} f\left(x_{i}\right) x_{i}\right\| \leqq M$ for all $n$. That is,

$$
\begin{aligned}
\sup _{\|f\| \leqq 1} & \left\|a_{1} f\left(x_{1}\right) e_{1}+\sum_{i=2}^{n} a_{i} f\left(x_{i}\right)\left(e_{i-1}-e_{i}\right)\right\| \\
& =\sup _{\|f\| \leqq 1}\left\{\left|a_{1} f\left(x_{1}\right)+a_{2} f\left(x_{2}\right)\right|+\sum_{i=2}^{n}\left|a_{i+1} f\left(x_{i+1}\right)-a_{i} f\left(x_{i}\right)\right|+\left|a_{n} f\left(x_{n}\right)\right|\right\} \leqq M .
\end{aligned}
$$

Let $f$ in $l^{\infty}$ be defined by $f=(1,1,0,0,1,1,0,0,1,1, \ldots)$. One easily checks that

$$
\begin{aligned}
\left|f\left(x_{i}\right)\right|=1 & \text { if } i \text { is odd, } \\
& =0 \quad \text { if } i \text { is even. }
\end{aligned}
$$

Hence since $\|f\|=1$ we have $\sum_{\sigma_{1}}\left|a_{i}\right| \leqq M$ (where $\sigma_{1}$ is the subset of $\{1,2, \ldots, n\}$ consisting of odd integers). Similarly, defining $g$ in $l^{\infty}$ by $g=(0,1,1,0,0,1,1, \ldots)$ we see

$$
\begin{aligned}
\left|g\left(x_{i}\right)\right| & =1 & & \text { if } i \text { is even, } \\
& =0 & & \text { if } i \text { is odd }
\end{aligned}
$$

so again $\sum_{\sigma_{2}}\left|a_{i}\right| \leqq M$ (where $\sigma_{2}$ is the set of even integers in $\{1,2, \ldots, n\}$ ). Since these results are independent of $n$, we then have $\sum_{i=1}^{\infty}\left|a_{i}\right| \leqq 2 M$ and it follows that $\left(x_{i} \otimes x_{i}\right)$ in $l^{1} \otimes_{\varepsilon} l^{1}$ is similar to $\left(e_{i}\right)$ in $l^{1}$.

As we have mentioned, the tensor product of unconditional bases need not be unconditional. However, in special cases the tensor product basis is always unconditional. 
Proposition 3.14. Let $\left(x_{i}\right)$ be an unconditional basis for $X$ and $\left(e_{i}\right)$ the unit vector basis for $c_{0}$. Then the tensor product basis $\left(x_{i} \otimes e_{j}\right)$ is unconditional in $X \otimes_{\varepsilon} c_{0}$.

Proof. Suppose $\sum a_{i j} x_{i} \otimes e_{j}$ converges in $X \otimes_{\varepsilon} c_{0}$. Then given $\delta>0$ there is a pair $\left(i_{0}, j_{0}\right)$ such that for $\left(i_{1}, j_{1}\right) \geqq\left(i_{0}, j_{0}\right)$ (in the tensor product ordering of these pairs),

$$
\left\|\sum_{\left(i_{0}, j_{0}\right)}^{\left(i_{1}, j_{1}\right)} a_{i j} x_{i} \otimes e_{j}\right\|<\frac{\delta}{K}
$$

(where $K$ is given by the $K$-condition for $\left(x_{i}\right)$ ).

By definition of the $\varepsilon$-norm we have, grouping together those tensors $a_{i j} x_{i} \otimes e_{j}$ having the same $j$ index and summing over the designated indices,

$$
\sup _{\|f\| \leqq 1}\left\|\sum_{j}\left(\sum_{i} a_{i j} f\left(x_{i}\right)\right) e_{j}\right\|<\frac{\delta}{K} \text {. }
$$

That is, $\sup _{j}\left\|\sum_{i} a_{i j} x_{i}\right\|<\delta / K$. Now if $\left(b_{i j}\right)$ is a 0,1 sequence then in exactly the same way

$$
\left\|\sum_{\left(i_{0}, j_{0}\right)}^{\left(i_{1}, j_{1}\right)} b_{i j} a_{i j} x_{i} \otimes e_{j}\right\|=\sup _{j}\left\|\sum_{i} b_{i j} a_{i j} x_{i}\right\| \leqq \sup _{j} K\left\|\sum_{i} a_{i j} x_{i}\right\|<K \cdot \frac{\delta}{K}=\delta,
$$

and $\left(x_{i} \otimes e_{j}\right)$ is unconditional.

We have used repeatedly the theorem of Gelbaum and de Lamadrid that if $\left(x_{i}\right)$ and $\left(y_{i}\right)$ are bases for $X$ and $Y$ then $\left(x_{i} \otimes y_{j}\right)$ is a basis for $X \otimes_{\varepsilon} Y$ and for $X \otimes_{\pi} Y$. If $\left(x_{i}\right)$ and $\left(y_{i}\right)$ are only assumed to be basic sequences in $X$ and $Y$ it is natural to ask whether $\left(x_{i} \otimes y_{j}\right)$ is basic in $X \otimes_{\varepsilon} Y$ and $X \otimes_{\pi} Y$.

In the case $X \otimes_{\varepsilon} Y$ the question is easily answered since $\left(x_{i}\right)$ is a basis for $\left[x_{i}\right]$, $\left(y_{i}\right)$ is a basis for $\left[y_{i}\right]$, and so $\left(x_{i} \otimes y_{j}\right)$ is a basis for $\left[x_{i}\right] \otimes_{\varepsilon}\left[y_{i}\right]$, a closed subspace of $X \otimes_{\varepsilon} Y$.

In connection with this idea the next proposition is of interest.

Proposition 3.15. Let $\left(x_{i}\right)$ be a basic sequence in $X$ and let $\left(y_{i}\right)$ be any sequence of nonzero elements of $Y$. Then the sequence $\left(x_{i} \otimes y_{i}\right)$ in $X \otimes_{\varepsilon} Y$ is a basic sequence.

Proof. Let $g$ be in $Y^{*}$ and $\|g\| \leqq 1$. Then there is a $K \geqq 1$ such that

$$
\left\|\sum_{i=1}^{n} a_{i} g\left(y_{i}\right) x_{i}\right\| \leqq K\left\|\sum_{i=1}^{n+p} a_{i} g\left(y_{i}\right) x_{i}\right\| \leqq K \sup _{\|g\| \leqq 1}\left\|\sum_{i=1}^{n+p} a_{i} g\left(y_{i}\right) x_{i}\right\|=K\left\|\sum_{i=1}^{n+p} a_{i} y_{i} \otimes x_{i}\right\| \text {. }
$$

Hence

$$
\left\|\sum_{i=1}^{n} a_{i} y_{i} \otimes x_{i}\right\| \leqq K\left\|\sum_{i=1}^{n+p} a_{i} y_{i} \otimes x_{i}\right\|
$$

and the proposition is proved.

If $\left(x_{i}\right)$ is a monotone basis for $X$, then the $K$ of Proposition 3.15 is 1 and we have

Corollary 3.16. Let $\left(x_{i}\right)$ be a monotone basis for $X$ and $\left(y_{i}\right)$ a basis for $Y$. Then the tensor diagonal $\left(x_{i} \otimes y_{i}\right)$ is monotone in $X \otimes_{\varepsilon} Y$. 
In contrast to Corollary 3.16 we show that the tensor product of monotone bases need not be monotone.

Recall that if $M$ and $N$ are compact metric spaces then

$$
C(M) \otimes_{\varepsilon} C(N)=C(M \times N)
$$

and $\left\|\sum_{i=1}^{n} z_{i} \otimes w_{i}\right\|=\sup _{(s, t)}\left|\sum_{i=1}^{n} z_{i}(s) w_{i}(t)\right|[2]$.

EXAmple 3.17. Let $\left(\phi_{i}\right)_{i=0}^{\infty}$ denote the usual Schauder basis for $C[0,1]$. One verifies easily that $\left\|\phi_{0} \otimes \phi_{0}+\phi_{0} \otimes \phi_{1}+\phi_{1} \otimes \phi_{1}\right\|=3$ while

$$
\left\|\phi_{0} \otimes \phi_{0}+\phi_{0} \otimes \phi_{1}+\phi_{1} \otimes \phi_{1}-\phi_{1} \otimes \phi_{0}\right\|=2 .
$$

Hence $\left(\phi_{i} \otimes \phi_{j}\right)$ is not monotone (although $\left(\phi_{i}\right)$ is monotone).

The proof of the next proposition makes use of the same ideas as that of Proposition 3.14 and will be omitted. The proposition itself should be compared with Proposition 3.14.

Proposition 3.18. Let $\left(x_{i}\right)$ be a monotone basis for $X$ and $\left(e_{i}\right)$ the unit vector basis for $c_{0}$. Then the basis $\left(x_{i} \otimes e_{j}\right)$ for $X \otimes_{\varepsilon} c_{0}$ is monotone.

REMARK 3.19. Joiner has shown, in a more general context than Banach spaces, that if $\left(x_{i}\right)$ and $\left(y_{i}\right)$ are both of type $\mathrm{P}\left(\right.$ type $\left.\mathrm{P}^{*}\right)$ then $\left(x_{i} \otimes y_{j}\right)$ is of type $\mathrm{P}$ (type $\mathrm{P}^{*}$ ) in $X \otimes_{\varepsilon} Y$ [13]. Since every subsequence of a type $\mathrm{P}^{*}$ basis is of type $\mathrm{P}^{*}$ it follows that the tensor diagonal of type $\mathrm{P}^{*}$ bases is of type $\mathrm{P}^{*}$. However this is in general not the case for bases of type $P$.

In fact, if $\left(x_{i}\right)$ is the basis of type $P$ for $C[0,1]$ constructed by Singer and Foiaş [7] then $x_{i}=\delta_{i} \phi_{i}$, where $\delta_{i}= \pm 1$ and $\left(\phi_{i}\right)$ is the usual Schauder basis for $C[0,1]$. However one can show that $\left(\phi_{i} \otimes \phi_{i}\right)$, and hence $\left(x_{i} \otimes x_{i}\right)$ is similar to the basis $\left(\phi_{i}^{2}\right)$ in $C[0,1][11]$ and this last is easily seen to be nontype $P$.

4. The $\pi$-tensor product. This section is concerned with the same general subject matter as $\$ 3$ except that we consider here the $\pi$-topology rather than the $\varepsilon$-topology. A number of the theorems proved in $\S 1$ for the space $X \otimes_{\varepsilon} Y$ have analogues in the space $X \otimes_{\pi} Y$. However, there is generally a strong contrast in the results obtained in these two topologies.

Our first result is a consequence of Theorem 3.4.

THEOREM 4.1. Let $\left(x_{i}\right)$ and $\left(y_{i}\right)$ be bases for $X$ and $Y$ respectively. Then the tensor product basis $\left(x_{i} \otimes y_{j}\right)$ for $X \otimes_{\pi} Y$ is boundedly complete if and only if $\left(x_{i}\right)$ and $\left(y_{i}\right)$ are boundedly complete.

Proof. The first implication is obvious from Lemmas 3.1 and 3.3.

If $\left(x_{i}, f_{i}\right)$ and $\left(y_{i}, g_{i}\right)$ are both boundedly complete then each of $\left(f_{i}\right)$ and $\left(g_{i}\right)$ are shrinking basic sequences. By Theorem 3.4, $\left(f_{i} \otimes g_{j}\right)$ is a shrinking basis for $\left[f_{i}\right] \otimes_{\varepsilon}\left[g_{i}\right]$, a closed subspace of $\left(X \otimes_{\pi} Y\right)^{*}$. Hence $\left(x_{i} \otimes y_{j}, f_{i} \otimes g_{j}\right)$ is boundedly complete. 
Simple examples show that both $\left(x_{i}\right)$ and $\left(y_{i}\right)$ may be of type $\mathrm{wc}_{0}$ and yet $\left(x_{i} \otimes y_{i}\right)$ (hence certainly $\left(x_{i} \otimes y_{j}\right)$ ) may not be of type $\mathrm{wc}_{0}$ in $X \otimes_{\pi} Y$. However the next proposition follows immediately from Theorem 3.7.

Proposition 4.2. Let $\left(x_{i}, f_{i}\right)$ and $\left(y_{i}, g_{i}\right)$ be bases of type $\left(\mathrm{wc}_{0}\right)^{*}$ for $X$ and $Y$ respectively. Then the basis $\left(x_{i} \otimes y_{j}\right)$ for $X \otimes_{\pi} Y$ is of type $\left(\mathrm{wc}_{0}\right)^{*}$.

As we have remarked following Theorem 3.12, if $\left(x_{i}\right)$ is an unconditional basis for $X$ and $\left(y_{i}\right)$ is a basis for $Y$, then $\left(x_{i} \otimes y_{i}\right)$ is an unconditional basic sequence in $X \otimes_{\pi} Y$. The converse is false.

EXAMPLE 4.3. Let $\left(x_{i}\right)$ be the conditional basis for $l^{1}$ defined by $x_{1}=e_{1}, x_{n}=$ $e_{n-1}-e_{n}$ for $n=2,3, \ldots$ Then the tensor diagonal $\left(x_{i} \otimes x_{i}\right)$ in $l^{1} \otimes_{\pi} l^{1}$ is similar to $\left(e_{i}\right)$ in $l^{1}$.

Proof. Using the well-known result that in $l^{1} \otimes_{\pi} W\left\|\sum_{i=1}^{n} e_{i} \otimes w_{i}\right\|=\sum_{i=1}^{n}\left\|w_{i}\right\|$ [2], one easily computes that for $n \geqq 3$

$$
\left\|\sum_{i=1}^{n} a_{i} x_{i} \otimes x_{i}\right\|=\sum_{i=1}^{n-1}\left|a_{i}+a_{i+1}\right|+2 \sum_{i=2}^{n-1}\left|a_{i}\right|+3\left|a_{n}\right| .
$$

Hence if $\sum_{i} a_{i} x_{i} \otimes x_{i}$ converges, then $\sum_{i}\left|a_{i}\right|<+\infty$ and $\left(x_{i} \otimes x_{i}\right)$ is similar to $\left(e_{i}\right)$ in $l^{1}$.

As we have mentioned in $\S 3$ the tensor product of unconditional bases need not be unconditional in $X \otimes_{\pi} Y$. However we can show, in a manner analogous to that of Proposition 3.14, that in some cases the tensor product is always unconditional.

Proposition 4.4. Let $\left(x_{i}\right)$ be an unconditional basis for $X$ and $\left(e_{i}\right)$ the unit vector basis of $l^{1}$. Then $\left(x_{i} \otimes e_{j}\right)$ is an unconditional basis for $X \otimes_{\pi} l^{1}$.

Proof. Suppose $\sum a_{i j} x_{i} \otimes e_{j}$ converges in $X \otimes_{\pi} l^{1}$. Then given $\delta>0$ there is a pair $\left(i_{0}, j_{0}\right)$ such that if $\left(i_{1}, j_{1}\right) \geqq\left(i_{0}, j_{0}\right)$ (in the ordering on the indices from the tensor product basis) then

$$
\left\|\sum_{\left(i_{0}, j_{0}\right)}^{\left(i_{1}, j_{1}\right)} a_{i j} x_{i} \otimes e_{j}\right\|<\frac{\delta}{K}
$$

where $K$ is given by the $K$-condition on $\left(x_{i}\right)$. By grouping together those tensors with the same $e_{j}$ term in them and summing over the designated indices we may write

$$
\left\|\sum_{\left(i_{0}, j_{0}\right)}^{\left(i_{1}, j_{1}\right)} a_{i j} x_{i} \otimes e_{j}\right\|=\left\|\sum_{j}\left(\sum_{i} a_{i j} x_{i}\right) \otimes e_{j}\right\|=\sum_{j}\left\|\sum_{i} a_{i j} x_{i}\right\|<\frac{\delta}{K} .
$$

Now if $\left(b_{i j}\right)$ is any 0,1 sequence, then

$$
\left\|\sum_{\left(i_{0}, j_{0}\right)}^{\left(i_{1}, j_{1}\right)} b_{i j} a_{i j} x_{i} \otimes e_{j} .\right\|=\sum_{j}\left\|\sum_{i} b_{i j} a_{i j} x_{i}\right\| \leqq \sum_{j} K\left\|\sum_{i} a_{i j} x_{i}\right\|<K \cdot \frac{\delta}{K}=\delta .
$$


Hence $\sum b_{i j} a_{i j} x_{i} \otimes e_{j}$ converges and $\left(x_{i} \otimes e_{j}\right)$ is an unconditional basis for $X \otimes_{\pi} l^{1}$.

Our next result is an analogue of Proposition 3.15. However we will need to impose the additional conditions that $\left(x_{i}\right)$ be a basis rather than just a basic sequence and that $Y$ have a basis. These assumptions are probably superfluous.

Proposition 4.5. Let $\left(x_{i}\right)$ be a basis for $X$ and suppose $Y$ has a basis. Then for any nonzero sequence $\left(z_{i}\right)$ in $Y,\left(x_{i} \otimes z_{i}\right)$ is a basic sequence in $X \otimes_{\pi} Y$.

Proof. Let $\left(y_{i}\right)$ be a basis for $Y$ and suppose

$$
\begin{aligned}
\sum_{i=1}^{n+p} a_{i} x_{i} \otimes z_{i} & =\sum_{k=1}^{m} v_{k} \otimes w_{k}=\sum_{k=1}^{m}\left(\sum_{j} b_{j}^{(k)} x_{j}\right) \otimes\left(\sum_{l} c_{l}^{(k)} y_{l}\right) \\
& =\sum_{j} \sum_{l}\left(\sum_{k=1}^{m} b_{j}^{(k)} c_{l}^{(k)}\right) x_{j} \otimes y_{l} .
\end{aligned}
$$

Since

$$
\sum_{j=1}^{n+p} a_{j} x_{j} \otimes z_{j}=\sum_{j=1}^{n+p} a_{j} x_{j} \otimes\left(\sum_{l} d_{l}^{(j)} y_{l}\right)=\sum_{j=1}^{n+p} \sum_{l} a_{j} d_{l}^{(j)} x_{j} \otimes y_{l}
$$

and since $\left(x_{i} \otimes y_{j}\right)$ is a basis for $X \otimes_{\pi} Y$ we must have

$$
\sum_{j} \sum_{l}\left(\sum_{k=1}^{m} b_{j}^{(k)} c_{l}^{(k)}\right) x_{j} \otimes y_{l}=\sum_{j=1}^{n+p} \sum_{l}\left(\sum_{k=1}^{m} b_{j}^{(k)} c_{l}^{(k)}\right) x_{j} \otimes y_{l}
$$

and $\sum_{k=1}^{m} b_{j}^{(k)} c_{l}^{(k)}=a_{j} d_{l}^{(j)}$ for $j=1,2, \ldots, n+p$ and any $l=1,2, \ldots$

Now $\sum_{j=1}^{n} a_{j} x_{j} \otimes z_{j}=\sum_{j=1}^{n} \sum_{l} a_{j} d_{l}^{(j)} x_{j} \otimes y_{l}$, which by the above is equal to

$$
\sum_{j=1}^{n} \sum_{l}\left(\sum_{k=1}^{m} b_{j}^{(k)} c_{l}^{(k)}\right) x_{j} \otimes y_{l}=\sum_{k=1}^{m}\left(\sum_{j=1}^{n} b_{j}^{(k)} x_{j}\right) \otimes\left(\sum_{l} c_{l}^{(k)} y_{l}\right)=\sum_{k=1}^{m} v_{k}^{\prime} \otimes w_{k}
$$

where $v_{k}^{\prime}=\sum_{j=1}^{n} b_{j}^{(k)} x_{j}$. Now $\left\|v_{k}^{\prime}\right\| \leqq K\left\|v_{k}\right\|$ so $K \sum_{k=1}^{m}\left\|v_{k}\right\|\left\|w_{k}\right\| \geqq \sum_{k=1}^{m}\left\|v_{k}^{\prime}\right\|\left\|w_{k}\right\|$. Since this is true for every representation of $\sum_{i=1}^{n+p} a_{i} x_{i} \otimes z_{i}$, we have

$$
K\left\|\sum_{i=1}^{n+p} a_{i} x_{i} \otimes z_{i}\right\| \geqq\left\|\sum_{i=1}^{n} a_{i} x_{i} \otimes z_{i}\right\|
$$

and by the $K$-condition $\left(x_{i} \otimes z_{i}\right)$ is basic in $X \otimes_{\pi} Y$.

The proof of Proposition 4.5 shows that the $K$ corresponding to the basic sequence $\left(x_{i} \otimes z_{i}\right)$ is the same $K$ which corresponds to the basis $\left(x_{i}\right)$. Hence we have

Corollary 4.6. Let $\left(x_{i}\right)$ be a monotone basis for $X$ and $\left(y_{i}\right)$ any basis for $Y$. Then the tensor diagonal $\left(x_{i} \otimes y_{i}\right)$ in $X \otimes_{\pi} Y$ is monotone.

Here again, as in the case of the $\varepsilon$-topology, the tensor product of monotone bases may not be monotone.

ExAmple 4.7. Let $\left(x_{i}\right)$ be the (monotone) basis for $l^{1}$ defined in Example 4.3. Then $\left\|x_{1} \otimes x_{1}+x_{2} \otimes x_{2}\right\|=5$ while $\left\|x_{1} \otimes x_{1}+x_{2} \otimes x_{2}-x_{2} \otimes x_{1}\right\|=3$. Hence $\left(x_{i} \otimes x_{j}\right)$ is not monotone in $l^{1} \otimes_{\pi} l^{1}$. 
In contrast to this example we state the following result. The proof is omitted since it follows closely that of Proposition 4.4. The proposition should be compared with Propositions 4.4, 3.14, and 3.18.

Proposition 4.8. Let $\left(x_{i}\right)$ be a monotone basis for $X$ and $\left(e_{i}\right)$ the unit vector basis for $l^{1}$. Then $\left(x_{i} \otimes e_{j}\right)$ is a monotone basis for $X \otimes_{\pi} l^{1}$.

RemarK. In $\S 3$ we showed that if $\left(x_{i}\right)$ is a basic sequence in $X$ and $\left(y_{i}\right)$ is a basic sequence in $Y$ then $\left(x_{i} \otimes x_{j}\right)$ is a basic sequence in $X \otimes_{\varepsilon} Y$. However the method of proof is not applicable to the case $X \otimes_{\pi} Y$ since $\left[x_{i}\right] \otimes_{\pi}\left[y_{i}\right]$ need not be a closed subspace of $X \otimes_{\pi}, Y$.

Problem. If $\left(x_{i}\right)$ is a basic sequence in $X$ and $\left(y_{i}\right)$ is a basic sequence in $Y$, is $\left(x_{i} \otimes y_{j}\right)$ a basic sequence in $X \otimes_{\pi} Y$ ?

A negative answer to this problem would also answer negatively the unsolved problem of whether every basic sequence in a Banach space may be extended to a basis for the whole space. For, if $\left(x_{i}\right)$ and $\left(y_{i}\right)$ are basic in $X$ and $Y$ respectively and $\left(z_{n}\right)$ and $\left(w_{m}\right)$ are extensions of each which are bases for $X$ and $Y$, then $\left(x_{i} \otimes y_{j}\right)$ is basic in $X \otimes_{\pi} Y$ being a subsequence of the basis $\left(z_{n} \otimes w_{m}\right)$ for $X \otimes_{\pi} Y$.

5. Properties of the tensor diagonal. As the results of this section show, a consideration of the tensor diagonal $\left(x_{i} \otimes y_{i}\right)$ of a tensor product basis is often useful in determining properties of the spaces $X \otimes_{\varepsilon} Y$ and $X \otimes_{\pi} Y$.

We will be concerned mainly with imposing conditions on the bases $\left(x_{i}\right)$ and $\left(y_{i}\right)$ so that the diagonal $\left(x_{i} \otimes y_{i}\right)$ in $X \otimes_{\varepsilon} Y$ or $X \otimes_{\pi} Y$ will be similar to some given basis and with characterizing the diagonals of certain well known bases.

Our first results in this direction also demonstrate additional permanence properties of tensor products of arbitrary bases with the unit vector bases of $c_{0}$ and $l^{1}$.

Proposition 5.1. Let $\left(x_{i}\right)$ be a basis for $X$ and $\left(e_{i}\right)$ the unit vector basis for $c_{0}$. Then the tensor diagonal $\left(x_{i} \otimes e_{i}\right)$ in $X \otimes_{\varepsilon} c_{0}$ is similar to $\left(e_{i}\right)$ in $c_{0}$.

Proof. Since $\left(e_{i}\right)$ in $c_{0}$ is unconditional, $\left(x_{i} \otimes e_{i}\right)$ in $X \otimes_{\varepsilon} c_{0}$ is unconditional by Theorem 3.12. By definition of the $\varepsilon$-norm,

$$
\left\|\sum_{i=1}^{n} x_{i} \otimes e_{i}\right\|=\sup _{\|f\| \leqq 1}\left\|\sum_{i=1}^{n} f\left(x_{i}\right) e_{i}\right\|=\sup _{1 \leqq i \leqq n} \sup _{\|f\| \leqq 1}\left|f\left(x_{i}\right)\right| \leqq \sup _{i}\left\|x_{i}\right\|<+\infty .
$$

Hence $\left(x_{i} \otimes e_{i}\right)$ is of type $\mathrm{P}$ and is therefore similar to $\left(e_{i}\right)$ in $c_{0}$.

A dual result is

Proposition 5.2. Let $\left(x_{i}\right)$ be a basis for $X$ and $\left(e_{i}\right)$ the unit vector basis for $l^{1}$. Then the tensor diagonal $\left(x_{i} \otimes e_{i}\right)$ in $X \otimes_{\pi} l^{1}$ is similar to $\left(e_{i}\right)$ in $l^{1}$.

Proof. If $\sum_{i} a_{i} x_{i} \otimes e_{i}$ converges in $X \otimes_{\pi} l^{1}$ then

$$
\left\|\sum_{i=m}^{n} a_{i} x_{i} \otimes e_{i}\right\|=\sum_{i=m}^{n}\left|a_{i}\right|\left\|x_{i}\right\| \stackrel{m, n}{\longrightarrow} 0 .
$$


Since $\inf _{i}\left\|x_{i}\right\|>0$ we must have $\sum_{i}\left|a_{i}\right|<+\infty$ and $\left(x_{i} \otimes e_{i}\right)$ is similar to $\left(e_{i}\right)$ in $l^{1}$.

We have shown in Theorem 3.12 that the tensor diagonal $\left(x_{i} \otimes y_{i}\right)$ of an unconditional basis $\left(x_{i}\right)$ for $X$ and a basis $\left(y_{i}\right)$ for $Y$ is unconditional. In the case where $\left(y_{i}\right)$ is of type $\mathrm{P}^{*}$ we can say much more.

Proposition 5.3. Let $\left(x_{i}\right)$ be an unconditional basis for $X$ and $\left(y_{i}\right)$ a basis of type P* for $Y$. Then the tensor diagonal $\left(x_{i} \otimes y_{i}\right)$ in $X \otimes_{\varepsilon} Y$ is similar to $\left(x_{i}\right)$.

Proof. If $\sum_{i} a_{i} x_{i} \otimes y_{i}$ converges in $X \otimes_{\varepsilon} Y$ then

$$
\sup _{\|g\| \geqq 1}\left\|\sum_{i=m}^{n} a_{i} g\left(y_{i}\right) x_{i}\right\| \stackrel{m, n}{\longrightarrow} 0 .
$$

Since $\left(y_{i}\right)$ is of type $\mathrm{P}^{*}$, there is a $g$ in $Y^{*}$ such that $\|g\|=1$ and $g\left(y_{i}\right)=b>0$ for all $i$. Hence by the above $\left\|\sum_{i=m}^{n} b a_{i} x_{i}\right\| \stackrel{m, n}{\longrightarrow} 0$, implying $\sum_{i} a_{i} x_{i}$ converges in $X$.

Conversely, if $\sum_{i} a_{i} x_{i}$ converges then since $\left(x_{i}\right)$ is unconditional, $\sum_{i} c_{i} a_{i} x_{i}$ converges uniformly for $\left(c_{i}\right)$ in any bounded subset of $l^{\infty}$ [14]. Since $\left\{\left(g\left(y_{i}\right)\right) \mid g\right.$ in $Y^{*}$, $\|g\| \leqq 1\}$ is a bounded subset of $l^{\infty}$, it follows that $\sum_{i} a_{i} g\left(y_{i}\right) x_{i}$ converges uniformly over $\|g\| \leqq 1$, and by definition of the $\varepsilon$-norm $\sum_{i} a_{i} x_{i} \otimes y_{i}$ converges in $X \otimes_{\varepsilon} Y$. Therefore $\left(x_{i}\right)$ is similar to $\left(x_{i} \otimes y_{i}\right)$.

Proposition 5.3 offers an interesting contrast between the $\varepsilon$ and $\pi$ topologies since an analogous proposition with $\varepsilon$ replaced by $\pi$ is not true.

ExAMPLE 5.4. Let $\left(e_{i}\right)$ denote the unit vector basis in $l^{p}(1<p<+\infty)$. Then $\left(e_{i}\right)$ in $l^{p}$ is unconditional while $\left(e_{i}\right)$ in $l^{1}$ is of type $\mathrm{P}^{*}$. However, by Proposition 5.2, $\left(e_{i} \otimes e_{i}\right)$ in $l^{p} \otimes_{\pi} l^{1}$ is similar to $\left(e_{i}\right)$ in $l^{1}$ (and hence not to $\left(e_{i}\right)$ in $l^{p}$ ).

Notice, however, that since $\|\cdot\|_{\varepsilon} \leqq\|\cdot\|_{\pi}$, it follows that if $\left(y_{i}\right)$ is of type $\mathrm{P}^{*}$ and $\sum_{i} a_{i} x_{i} \otimes y_{i}$ converges in $X \otimes_{\pi} Y$, then $\sum_{i} a_{i} x_{i}$ converges in $X$.

The next theorem provides an interesting characterization of the tensor diagonal $\left(e_{i} \otimes e_{i}\right)$ in $l^{p} \otimes_{\varepsilon} l^{r}(1<p, r<\infty)$ where $\left(e_{i}\right)$ denotes the unit vector basis.

THEOREM 5.5. Let $1<p, r<+\infty$ and set $q=p /(p-1)$. Then

(i) If $r \geqq q$ the tensor diagonal $\left(e_{i} \otimes e_{i}\right)$ in $l^{p} \otimes_{\varepsilon} l^{r}$ is similar to $\left(e_{i}\right)$ in $c_{0}$.

(ii) If $r<q$ the tensor diagonal $\left(e_{i} \otimes e_{i}\right)$ in $l^{p} \otimes_{\varepsilon} l^{r}$ is similar to $\left(e_{i}\right)$ in $l^{a r /(q-r)}$.

Proof. (i) By Theorem 3.13, $\left(e_{i} \otimes e_{i}\right)$ in $l^{p} \otimes_{\varepsilon} l^{r}$ is unconditional. Now

$$
\left\|\sum_{i=1}^{n} e_{i} \otimes e_{i}\right\|=\sup _{\left\|x^{*}\right\| \leqq 1 ; x^{*} \text { in } l^{q}}\left\|\sum_{i=1}^{n} x^{*}\left(e_{i}\right) e_{i}\right\|_{l^{r}} \leqq \sup _{\left\|x^{*}\right\| \leqq 1 ; x^{*} \text { in } l^{r}}\left\|\sum_{i} x^{*}\left(e_{i}\right) e_{i}\right\|_{l^{r}} \leqq 1
$$

(since $q \leqq r$ implies any $x^{*}$ in $l^{q}$ is in $l^{r}$ and $\left\|x^{*}\right\|_{l^{q}} \leqq 1$ implies $\left\|x^{*}\right\|_{l^{r}} \leqq 1$ ). Hence $\left(e_{i} \otimes e_{i}\right)$ is of type $\mathrm{P}$ and must then be similar to $\left(e_{i}\right)$ in $c_{0}$.

(ii) Suppose $r<q$ and $\sum a_{i} e_{i} \otimes e_{i}$ converges in $l^{p} \otimes_{\varepsilon} l^{r}$. Then

$$
\sup _{\left\|x^{*}\right\| \leqq 1 ; x^{*} \text { in } l^{a}}\left[\sum_{i=m}^{n}\left|a_{i}\right|^{r}\left|x^{*}\left(e_{i}\right)\right|^{r}\right]^{1 / r} \rightarrow 0,
$$

implying $\sum_{i}\left|a_{i}\right|^{r}\left|x^{*}\left(e_{i}\right)\right|^{r}$ converges for all $x^{*}$ in $l^{q}$. 
For any sequence $\left(b_{i}\right)$ in $l^{q / r}$ (note $q / r>1$ ), define $x^{*}\left(e_{i}\right)=\left|b_{i}\right|^{1 / r}$. Clearly the sequence $\left(x^{*}\left(e_{i}\right)\right)$ defined this way is an element of $l^{q}$ which we denote by $x^{*}$. By the above we then have

$$
\sum_{i}\left|a_{i}\right|^{r}\left(\left|b_{i}\right|^{1 / r}\right)^{r}=\sum_{i}\left|a_{i}\right|^{r}\left|b_{i}\right|
$$

converges for all $\left(b_{i}\right)$ in $l^{q / r}$, and by a well known result it follows that $\left|a_{i}\right|^{r}$ is in $l^{q /(q-r)}=\left(l^{q / r}\right)^{*}$. That is, $\left(a_{i}\right)$ is in $l^{q r /(q-r)}$.

Conversely, if $\left(a_{i}\right)$ is in $l^{q r /(q-r)}$, then for any $m, n$

$$
\begin{aligned}
\sup _{\left\|x^{*}\right\| \leqq 1: x^{*} \text { in } l^{q}}\left[\sum_{i=m}^{n}\left|a_{i}\right|^{r}\left|x^{*}\left(e_{i}\right)\right|^{r}\right]^{1 / r} & \leqq \sup _{\left\|x^{*}\right\| \leqq 1}\left[\left\{\sum_{i=m}^{n}\left(\left|a_{i}\right|^{r}\right)^{q /(q-r)}\right\}^{(q-r) / q} \cdot\left\{\sum_{i=m}^{n}\left(\left|x^{*}\left(e_{i}\right)\right|^{r}\right)^{q / r}\right\}^{r / q}\right]^{1 / r}
\end{aligned}
$$

by Hölder's inequality. But this last is equal to

$$
\sup _{\left\|x^{*}\right\| \leqq 1}\left[\sum_{i=m}^{n}\left|a_{i}\right|^{q r /(q-r)}\right]^{(q-r) / q r} \cdot\left[\sum_{i=m}^{n}\left|x^{*}\left(e_{i}\right)\right|^{q}\right]^{1 / q} \leqq\left[\sum_{i=m}^{n}\left|a_{i}\right|^{q r /(q-r)}\right]^{(q-r) / q r} \stackrel{m, n}{\longrightarrow} 0 .
$$

Hence if $\left(a_{i}\right)$ is in $l^{q r /(q-r)}$, then $\sum_{i} a_{i} e_{i} \otimes e_{i}$ converges in $l^{p} \otimes_{\varepsilon} l^{r}$ and we see $\left(e_{i} \otimes e_{i}\right)$ is similar to $\left(e_{i}\right)$ in $l^{q r /(q-r)}$.

NoTE. The special case $q=r$ of part (i) of Theorem 5.5 was proved by Dunford and Schatten [5].

Corollary 5.6. Given any $1<s<+\infty$ there is a $1<p<2$ such that $\left(e_{i} \otimes e_{i}\right)$ in $l^{p} \otimes_{\varepsilon} l^{p}$ is similar to $\left(e_{i}\right)$ in $l^{s}$.

Proof. For $1<s<+\infty$ set $q=2 s /(s-1)$. Then $q>2$ so $p=q /(q-1)<2$. By Theorem 5.5, $\left(e_{i} \otimes e_{i}\right)$ in $l^{p} \otimes_{\varepsilon} l^{p}$ is similar to $\left(e_{i}\right)$ in $l^{p q /(q-p)}$. It is easily seen that $p q /(q-p)=s$.

In order to describe the diagonals $\left(e_{i} \otimes e_{i}\right)$ in $l^{p} \otimes_{\pi} l^{r}$ we will need the following lemma which is useful in duality type proofs.

Lemma 5.7. Let $\left(x_{i}, f_{i}\right)$ be a basis for $X$ having a subsequence $\left(x_{n_{i}}\right)$ which is similar to $\left(e_{i}\right)$ in $c_{0}$. Then the corresponding subsequence $\left(f_{n_{i}}\right)$ of $\left(f_{i}\right)$ in $X^{*}$ is similar to $\left(e_{i}\right)$ in $l^{1}$.

Proof. The sequence $\left(x_{n_{i}}\right)$ is similar to $\left(e_{i}\right)$ in $c_{0}$ so the associated sequence of coefficient functionals $\left(g_{n_{1}}\right)$ in $\left[x_{n_{i}}\right]^{*}$ is similar to $\left(e_{i}\right)$ in $l^{1}$.

It is clear that $g_{n_{i}}=\left.f_{n_{i}}\right|_{\left[x_{\left.n_{i}\right]}\right.}$ for each $i$. Hence if $\sum_{i} a_{i} f_{n_{i}}$ converges, then $\left.\sum_{i} a_{i} f_{n_{i}}\right|_{\left[x_{n i}\right]}$ $=\sum_{i} a_{i} g_{n_{i}}$ converges. But then by the above $\sum_{i}\left|a_{i}\right|<+\infty$ and $\left(f_{n_{i}}\right)$, being seminormalized, is similar to $\left(e_{i}\right)$ in $l^{1}$.

NoTE. It follows from a previous comment we have made (\$2) about $\left[f_{i}\right]$ being norm-determining over $X$ that if $\left(x_{i}, f_{i}\right)$ is a basis for $X$ such that $\left(f_{n_{i}}\right)$ is similar to $\left(e_{i}\right)$ in $c_{0}$, then $\left(x_{n_{i}}\right)$ is similar to $\left(e_{i}\right)$ in $l^{1}$. 
Corollary 5.8. Let $1<m, n<+\infty$ and $n /(n-1) \geqq m$. Then $\left(e_{i} \otimes e_{i}\right)$ in $l^{m} \otimes_{\pi} l^{n}$ is similar to $\left(e_{i}\right)$ in $l^{1}$.

Proof. Since $n /(n-1) \geqq m$ it follows from Theorem 5.5 that $\left(e_{i} \otimes e_{i}\right)$ in $l^{m /(m-1)}$ $\otimes_{\varepsilon} l^{n /(n-1)}$ is similar to $\left(e_{i}\right)$ in $c_{0}$. Hence by Lemma $5.7\left(e_{i} \otimes e_{i}\right)$ in $l^{m} \otimes_{\pi} l^{n}$ $=\left(l^{m /(m-1)} \otimes_{\varepsilon} l^{n /(n-1)}\right)^{*}$ is similar to $\left(e_{i}\right)$ in $l^{1}$.

It is a trivial corollary of Theorem 5.5 that $\left(e_{i} \otimes e_{i}\right)$ in $l^{p} \otimes_{\varepsilon} l^{q}\left(p^{-1}+q^{-1}=1\right)$ is similar to $\left(e_{i}\right)$ in $c_{0}$. The next theorem shows this is only a special case of a more general result.

THEOREM 5.9. Let $X$ be a space with an unconditional basis $\left(x_{i}, f_{i}\right)$. Then the tensor diagonal $\left(x_{i} \otimes f_{i}\right)$ in $X \otimes_{\varepsilon}\left[f_{i}\right]$ is similar to $\left(e_{i}\right)$ in $c_{0}$.

Proof. By Theorem 3.12 the diagonal $\left(x_{i} \otimes f_{i}\right)$ is unconditional. We show it is also of type $\mathrm{P}$ and hence similar to $\left(e_{i}\right)$ in $c_{0}$.

By definition

$$
\left\|\sum_{i=1}^{n} x_{i} \otimes f_{i}\right\|=\sup _{\|x\| \leqq 1}\left\|\sum_{i=1}^{n} f_{i}(x) x_{i}\right\| \leqq K \sup _{\|x\| \leqq 1}\left\|\sum_{i} f_{i}(x) x_{i}\right\|=K
$$

(where $K$ is given by the $K$-condition on $\left(x_{i}\right)$ ). Hence $\left(x_{i} \otimes f_{i}\right)$ is of type $\mathrm{P}$ in $X \otimes_{\varepsilon}\left[f_{i}\right]$.

COROLlaRY 5.10. Let $X$ be a space with an unconditional basic sequence $\left(y_{i}\right)$ such that there is a continuous linear mapping from $X$ onto $\left[y_{i}\right]\left(\right.$ e.g. if $\left[y_{i}\right]=X$ or $\left[y_{i}\right]$ is complemented in $X$ ). Then $c_{0}$ can be embedded in $X \otimes_{\varepsilon} X^{*}$.

Proof. Suppose $T: X \rightarrow\left[y_{i}\right]$ is a continuous linear mapping which is onto. Then $\left[y_{i}\right]=X / K$ where $K$ is the kernel of $T$. It follows that $\left[y_{i}\right]^{*}$ can be embedded in $X^{*}$ (since $\left[y_{i}\right]^{*}=[X / K]^{*}=K^{0} \subset X^{*}[18$, p. 161]). The conclusion now follows from Theorem 5.9 since $\left[y_{i}\right] \otimes_{\varepsilon}\left[y_{i}\right]^{*}$ is a closed subspace of $X \otimes_{\varepsilon} X^{*}$.

COROLlary 5.11. Let $X$ be a space with an unconditional basis. Then $X \otimes_{\varepsilon} X^{*}$ is not weakly complete.

COROLlary 5.12. Let $X$ be a reflexive space with an unconditional basis. Then $X \otimes_{\varepsilon} X^{*}$ has a basis which is neither shrinking nor boundedly complete.

Proof. If $\left(x_{i}, f_{i}\right)$ is an unconditional basis for $X$ then $\left(x_{i} \otimes f_{j}\right)$ is a basis for $X \otimes_{\varepsilon} X^{*}$ whose diagonal is similar to $\left(e_{i}\right)$ in $c_{0}$. Since no separable conjugate space contains $c_{0}$ [1], the space $X \otimes_{\varepsilon} X^{*}$ has no boundedly complete basis.

If every basis were shrinking then $X \otimes_{\varepsilon} X^{*}$ would be reflexive [22], contradicting the fact that $c_{0} \subset X \otimes_{\varepsilon} X^{*}$.

Corollary 5.13. Let $X$ be a reflexive space with an unconditional basis. Then $X \otimes_{\varepsilon} X^{*}$ is not quasi-reflexive of order $n$ for any $n \geqq 0$. 
Proof. Every closed subspace of a quasi-reflexive space is quasi-reflexive [3]. Since $c_{0}$ is not quasi-reflexive, the result follows immediately from Theorem 5.9. A dual result to Theorem 5.9 is

Corollary 5.14. Let $\left(x_{i}, f_{i}\right)$ be an unconditional basis for $X$. Then $\left(x_{i} \otimes f_{i}\right)$ in $X \otimes_{\pi}\left[f_{i}\right]$ is similar to $\left(e_{i}\right)$ in $l^{1}$.

Proof. By Theorem 3.12 the diagonal $\left(x_{i} \otimes f_{i}\right)$ in $X \otimes_{\pi}\left[f_{i}\right]$ is unconditional. Also, we have previously seen that the coefficient functionals associated with the basic sequence $\left(x_{i} \otimes f_{i}\right)$ in $X \otimes_{\pi}\left[f_{i}\right]$ are just the restrictions to $\left[x_{i} \otimes f_{i}\right]$ of the functionals $\left(f_{i} \otimes x_{i}\right)$ in $\left[f_{i}\right] \otimes_{\varepsilon}\left[x_{i}\right] \subset\left(X \otimes_{\pi}\left[f_{i}\right]\right)^{*}$.

Now $\left(f_{i} \otimes x_{i}\right)$ in $\left[f_{i}\right] \otimes_{\varepsilon}\left[x_{i}\right]$ is similar to $\left(e_{i}\right)$ in $c_{0}$ by Theorem 5.9, and hence is of type P. It is clear that the restrictions of a type $P$ basic sequence will also be of type $\mathrm{P}$. Therefore the coefficient functionals of the basic sequence $\left(x_{i} \otimes f_{i}\right)$ are of type $\mathrm{P}$, implying $\left(x_{i} \otimes f_{i}\right)$ is of type $\mathrm{P}^{*}$ and hence similar to $\left(e_{i}\right)$ in $l^{1}$.

From Corollary 5.14 we obtain the following interesting result.

Corollary 5.15. Let $X$ be a reflexive space with an unconditional basis. Then $\mathscr{L}(X, X)$ is nonseparable.

Proof. If $\left(x_{i}\right)$ is unconditional, then, by Corollary 5.14, $\left(x_{i} \otimes f_{i}\right)$ in $X \otimes_{\pi} X^{*}$ is similar to $\left(e_{i}\right)$ in $l^{1}$. Therefore $l^{\infty}$ is isomorphic to a factor space of $\left(X \otimes_{\pi} X^{*}\right)^{*}$ $=\mathscr{L}\left(X, X^{* *}\right)=\mathscr{L}(X, X)$ and the conclusion follows.

\section{REFERENCES}

1. C. Bessaga and A. Pelczyński, On bases and unconditional convergence of series in Banach spaces, Studia Math. 17 (1958), 151-164. MR 22 \#5872.

2. C. Bessaga and J. R. Retherford, Lectures on nuclear spaces and related topics, Lecture Notes, Louisiana State University, Baton Rouge, La., rev. version (to appear).

3. P. Civin and B. Yood, Quasi-reflexive spaces, Proc. Amer. Math. Soc. 8 (1957), 906-911. MR 19 \#756.

4. M. M. Day, Normed linear spaces, 2nd ed., Ergebnisse der math. und ihrer Grenzgebiete, Heft 21, Academic Press, New York and Springer-Verlag, Berlin and New York, 1962. MR 26 \#2847.

5. N. Dunford and R. Schatten, On the associate and conjugate space for the direct product of Banach spaces, Trans. Amer. Math. Soc. 59 (1946), 430-436. MR 7, 455.

6. N. Dunford and J. T. Schwartz, Linear operators. I: General theory. Pure and Appl. Math., vol. 7, Interscience, New York, 1958. MR 22 \#8302.

7. C. Foiaş and I. Singer, On bases in $C[0,1]$ and $L^{1}([0,1])$, Rev. Roumaine Math. Pures Appl. 10 (1965), 931-960. MR 34 \#6509.

8. B. R. Gelbaum and J. Gil de Lamadrid, Bases of tensor products of Banach spaces, Pacific J. Math. 11 (1961), 1281-1286. MR 26 \#5394.

9. M. M. Grinblyum, On the representation of a space of the type $B$ in the form of a direct sum of subspaces, Dokl. Akad. Nauk SSSR 70 (1950), 749-752. (Russian) MR 11, 525.

10. A. Grothendieck, Produits tensoriels topologiques et espaces nucléaires, Mem. Amer. Math. Soc. No. 16 (1955). MR 17, 763. 
11. J. R. Holub, Tensor product bases and tensor diagonals, Ph.D. Dissertation, Louisiana State University, Baton Rouge, La., 1969.

12. J. R. Holub and J. R. Retherford, Some curious bases for $c_{0}$ and $C[0,1]$, Studia Math. 34 (1970), 227-240.

13. H. F. Joiner, Schauder bases and topological tensor products, Dissertation, Florida State University, Tallahassee, Fla., 1968.

14. C. W. McArthur and J. R. Retherford, Some applications of an inequality in locally convex spaces, Trans. Amer. Math. Soc. 137 (1969), 115-123. MR 39 \#748.

15. A. Pełczyński and W. Szlenk, An example of a non-shrinking basis, Rev. Roumaine Math. Pures Appl. 10 (1965), 961-966. MR 34 \#3284.

16. J. R. Retherford, Shrinking bases in Banach spaces, Amer. Math. Monthly 73 (1966), 841-846. MR 34 \#1831.

17. _- A semishrinking basis which is not shrinking, Proc. Amer. Math. Soc. 19 (1968), 766. MR 37 \#739.

18. H. H. Schaefer, Topological vector spaces, Macmillan, New York, 1966. MR 33 \#1689.

19. R. Schatten, A theory of cross-spaces, Ann. of Math. Studies, no. 26, Princeton Univ. Press, Princeton, N. J., 1950. MR 12, 186.

20. I. Singer, Basic sequences and reflexivity of Banach spaces, Studia Math. 21 (1961/62), 351-369. MR 26 \#4155.

21. —_, Weak* bases in conjugate Banach spaces. II, Rev. Math. Pures Appl. (Bucarest) 8 (1963), 575-584. MR 31 \#2600.

22. M. Zippin, A remark on bases and reflexivity in Banach spaces, Israel J. Math. 6 (1968), 74-79. MR 38 \#4972.

Louisiana State University,

Baton Rouge, Louisiana 70803

Virginia Polytechnic Institute,

BlaCKSBURG, Virginia 24061 